\title{
Ein runder Tisch für den Wirtschaftsverkehr Prozessuale Planungs- und Kommunikationsformen
}

Nicht nur in der Verkehrsplanung werden zunehmend «intermediäre» Einrichtungen wie Runde Tische, Moderationsund Konfliktforen eingesetzt, sei es zur Beschleunigung vermeintlich "langwieriger" Genehmigungsverfahren, sei es zur Verständigung zwischen verschiedenen Akteuren und zum Abgleich divergierender Interessen, sei es zur Förderung von Kooperation. Auf die mit solchen neven Politikformen allgemein verbundenen Chancen und Risiken soll hier nicht näher eingegangen werden (vgl. Selle 1992). An dieser Stelle wird vielmehr ein Überblick über praktische Erfahrungen gegeben, die mit einer "Güterverkehrsrunde" im Forschungsvorhaben "Stadtverträglicher Wirtschaftsverkehr im Bergischen Städtedreieck» gemacht wurden (vgl. Hesse 1994). Beim Wirtschaftsverkehr handelt es sich um Gütertransporte und Dienstleistungsverkehre, die in grossen Städten einen Anteil von bis zu 10\% (Güterverkehr mit Lastfahrzeugen) bzw. 20\% $(\mathrm{Pkw})$ des Gesamtverkehrs haben. Diese Verkehrsart ist durch starke Zuwachsraten und grossen Handlungsdruck aufgrund der spezifischen Belastungen durch den Lkw-Verkehr geprägt. Transport und Logistik wirken sehr dynamisch auf die Stadtentwicklung ein.

In vielen Städten wird daher in jüngster Zeit an der Entwicklung von Konzepten für den Güterverkehr gearbeitet, vielfach werden in diesem Kontext auch Diskussionskreise mit privaten und öffentlichen Akteuren eingerichtet. Dafür spricht, dass der Wirtschaftsverkehr viel stärker als der Personenverkehr Ausdruck und Ergebnis unternehmerischen Handelns ist. Eine entsprechende Sensibilisierung der Planung für betriebliche Prozesse ist daher sinnvoll, wie umgekehrt auch Unternehmen von der Problemsicht öffentlicher Akteure lernen können. Wir gehen davon aus, dass der Austausch zwischen Städtebau und Unternehmen nicht nur das Verständnis der jeweils anderen Position fördert, sondern unter optimalen Bedingungen auch gemeinsame Lösungen möglich macht. Das im Rahmen des Modellvorhabens genutzte Instrument der Güterverkehrsrunde hat sich als geeignet erwiesen, ein solches Kommunikations- und Konfliktmanagement zu organisieren.

\section{Das Konfliktmilieu von Städtebau und Wirtschaftsverkehr}

Vielerorts gestaltet sich das Verhältnis der städtebaulichen Planung zu den Unternehmen problematisch. Dies hat im vorliegenden Fall mehrere Gründe. Zum einen liegen die Konflikte zwischen Städtebau und Wirtschaftsverkehr in der spezifischen Wachstumsdynamik des Gütertransportes. Zunehmend häufige, bedarfsorientierte Lieferungen, wachsende Gewichte und Achslasten von Volumenfahrzeugen und die anhaltend hohe Flächennachfrage im Bereich Transport, Umschlag, Lagerei üben einen starken Druck auf die gewachsenen Stadtstrukturen und städtischen Nutzungssysteme aus. Der Wirtschaftsverkehr beeinträchtigt mit seinen Lärm- und Luftschadstoffemissionen die Wohn-, Aufenthalts- und Umfeldqualität der Stadt; er beeinflusst aber auch die Qualität des Wirtschaftsstandorts. Diesbezüglich ist die Bedeutung des harten Standortfaktors Verkehrsinfrastruktur nicht rückläufig, sondern hat - von der öffentlichen Diskussion über die Folgen des Verkehrswachstums unberührt eher noch zugenommen (Verkehrsabhängigkeit der Produktion). Dies gilt auch dann, wenn Standortentscheidun- gen der Betriebe verstärkł nach Umweltqualität, Wohnumfeldkriterien etc. getroffen werden.

Zweitens passen die heutigen Anforderungen an die (Verkehrs-)Infrastruktur nicht mehr in die traditionellen städtebaulichen Strukturen. Ginge es primär nach den logistischen Erfordernissen der Ökonomie (Dimensionierung und Zugänglichkeit der Infrastruktur), müssten die Städte heute völlig neu geplant und gebaut werden, den spezifischen $\mathrm{Di}$ mensionsmassen der Transportgefässe folgend. Diese Umbruch-Logik hat, betrachtet man ihre impliziten Zwänge zur Aufweitung von Nutzungszeiten, zur Bereitstellung der notwendigen Flächen, zur Funktionstrennung statt zur Funktionsmischung - durchaus den Charakter «struktureller Gewalt». Offenbar zählt nicht mehr die Qualität eines Raumes "an sich", sondern die Maxime seiner möglichst reibungslosen Versorgung.

Drittens ist das Problemfeld Städtebau und Wirtschaftsverkehr durch eine unterentwickelte Fähigkeit der Akteure zur Verständigung und zur Austragung von Konflikten gekennzeichnet. Während die Organisation der politischen Interessen des Güter- und Wirtschaftsverkehrs sehr effizient und durchsetzungsstark ist, ist es bisher nicht gelungen, im Umfeld der Logistik eine entsprechende Lobby für städtebauliche Interessen zu organisie-

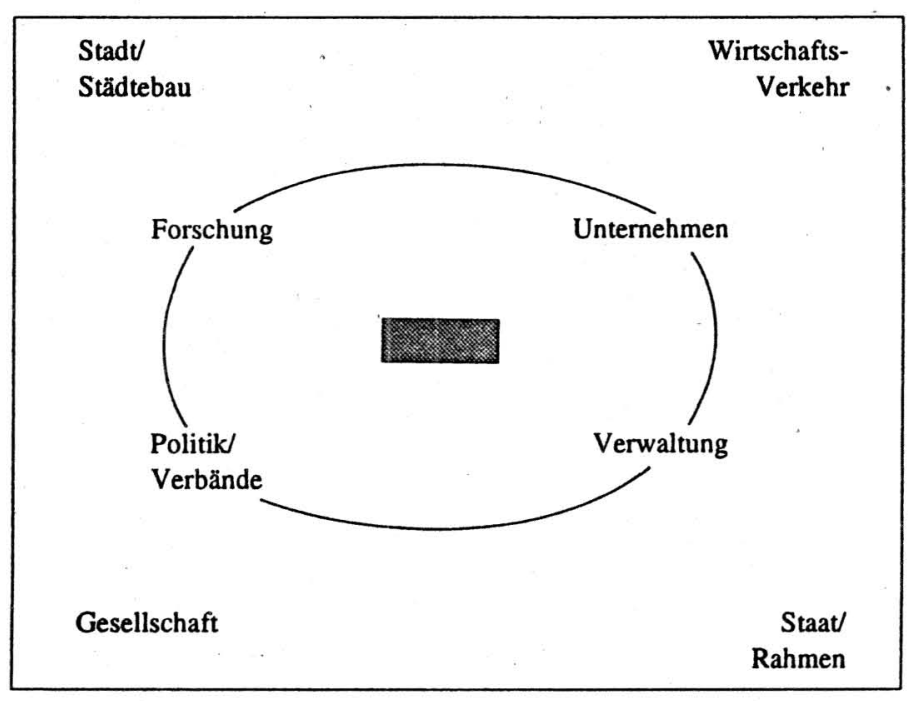

Abb. 1: Akteurssystem des Wirtschaftsverkehrs 
ren, zumal die Kommunen als Trägerinnen der Bauleitplanung und Flächennutzung immer weniger die Rolle des Widerlagers ausüben wollen oder können. Je schwieriger sich mit Blick auf die Verkehrssituation vor Ort die Leistungserstellung für die Unternehmen darstellt, um so problematischer entwickelt sich das Milieu von den Voraussetzungen für einen vorurteilsfreien Diskurs. Zwischen beiden Handlungs- und Akteursebenen findet kein nennenswerter Austausch statt; beide Seiten wissen relativ wenig von den Motiven, Problemen und Restriktionen der jeweils anderen.

Schliesslich trägt auch die politische Entscheidungssituation zu verstärkten Unstimmigkeiten bei: Beide Akteursgruppen bewegen sich in einem gesellschaftlichen Kontext, der politische Entscheidungen im Sinne von Politik als Regelung der öffentlichen Ordnung und Aushandlung von Konflikten zunehmend durch die Bedienung von Partikularinteressen ersetzt. Dies führt zum einen dazu, dass nur diejenigen Bedürfnisse und Ansprüche wahrgenommen werden, die auch über entsprechend effektvolle Artikulationsmöglichkeiten verfügen; zum anderen trägt diese Entwicklung tendenziell zu einer Politikunfähigkeit der Gesellschaft i.w.S. bei, die der gestiegenen Problemdichte, dem sozial-ökologischen Handlungsbedarf und der wachsenden Komplexität der Verhältnisse nicht mehr gerecht wird. Schliesslich werden die ökologischen und städtebaulichen Folgeprobleme unternehmerischen Handelns in Zeiten der Rezession häufig verdrängt, was die Problemlösungskompetenz der örtlichen Akteure nicht gerade erhöht.

\section{Ziele, Aufgaben, Anlässe}

Ziel der Güterverkehrsrunde im Rahmen des Modellvorhabens «Stadtverträglicher Wirtschaftsverkehr» war es, den als notwendig anerkannten Austausch der am Güter- und Wirtschaftsverkehr Beteiligten, der vorher nur informell bestand, zu verstetigen, vor allem zwischen Unternehmen und Städtebau (Kommunen) sowie unter den Unternehmen. Dies ist nach drei Jahren Arbeit in grosser Runde und in Arbeitsgruppen gelungen, wenn

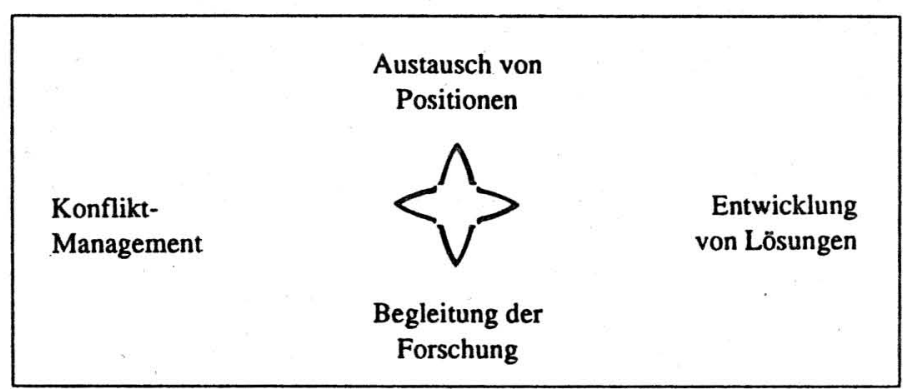

Abb. 2: Zielsystem der Güterverkehrsrunde

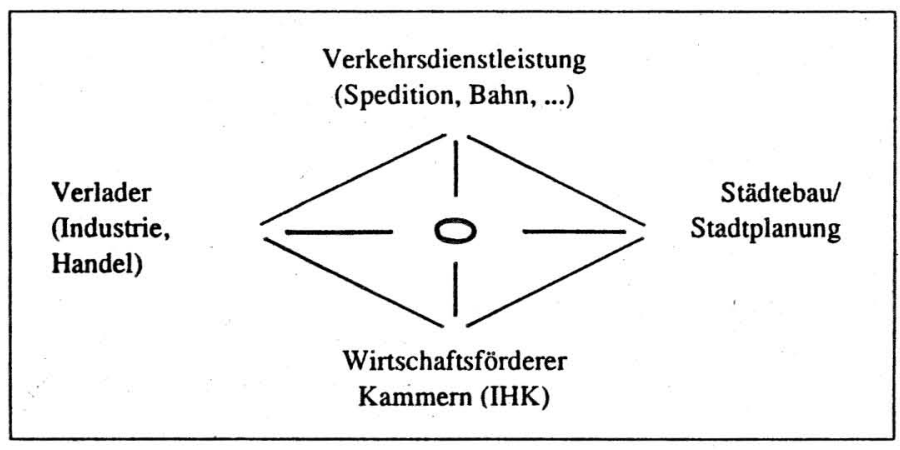

Abb. 3: Beteiligte der Güterverkehrsrunde

auch die Beteiligung und Zusammensetzung der einzelnen Sitzungen sehr unterschiedlich waren.

Aufgaben der Runde waren die kritische Begleitung der Arbeiten des Modellvorhabens, die Diskussion von Ergebnissen hinsichtlich Plausibilität und Umsetzbarkeit sowie das Einbringen von Ideen, Leistungen und Problemlösungspotentialen der einzelnen Akteure. Die Güterverkehrsrunde sollte Bereitschaft zum Austausch von Meinungen wecken, Entscheidungen und ihr konkretes Bedingungsgefüge transparent machen und Spielräume für konsensfähige Lösungen ausloten. Auch dies stellt natürlich hohe Anforderungen an die beteiligten Akteure. Der Städtebau hat dabei nicht die Rolle des allgemeinen Bedenkenträgers, sondern er soll innovativen Ideen zur Umsetzung verhelfen. Gleichwohl bringt das Ziel, die Gemeinwohlinteressen nicht aus dem Blickfeld zu verlieren, die kommunale Politik schnell in die unternehmerische Kritik - vor allem dann, wenn sie ihre Anliegen offensiv vertritt.

Um mit dieser Kommunikationsstrategie zu neuen Formen der Regulation konkurrierender Nutzungsansprüche zu kommen, müssen bestimmte Voraussetzungen erfüllt sein; deren Kenntnis kann auch dazu beitragen, dass solche Aktivitäten nicht in unverbindlichen $\mathrm{Ge}$ sprächskreisen enden. Zur Einrichtung einer Güterverkehrsrunde bedarf es nach unseren Erfahrungen zweier wichtiger "Incentives»: Zum einen konkreter Probleme vor Ort (z. B. Engpässe im Verkehrsnetz, über das "normale» Mass hinaus), zum anderen gewichtiger Einzelplanungen an relevanten Standorten, die von überbetrieblicher bzw. öffentlicher Bedeutung sind und über die planungsrechtliche Behandlung hinaus eine intensive Abstimmung zwischen Kommune und Unternehmung als sinnvoll erscheinen lassen. Nur unter diesen Voraussetzungen besteht bei den Unternehmen Veranlassung bzw. Bereitschaft, sich auf das Experiment einer Mitwirkung in solchen Güterverkehrskreisen einzulassen, ggf. auch über einen längeren Zeitraum.

\section{Zusammensetzung der Güterverkehrsrunde}

Eine Güterverkehrsrunde sollte nicht den Anspruch verfolgen, die Gesamtheit der im städtischen Güterverkehr vertretenen Interessen in Quantität und Qualität abdecken zu wollen. Sie wird nie repräsentativ für eine Stadt, Region oder Branche sein können. Die Runde sollte aber möglichst relevante und an der Sache persönlich interessierte Akteure aus der Region zusammenbringen. Als Zufallsauslese und um arbeitsfähig zu bleiben, ist notwendigerweise eine relativ kleine Gruppe sinnvoll, etwa von 35-40 Personen. Voraussetzung für das zielorientierte Agieren ist eine überschaubare Zahl von Beteiligten, je nach den lokalen und regionalen Strukturen sollte ein guter Mix aus verschiedenen Strukturen (Branchen, Betriebsgrössen, «alte» Betriebe, moderne Unternehmen) angestrebt werden. 
In der "Güterverkehrsrunde Bergisches Städtedreieck» haben Vertreter aus Industrie- und Handelsbetrieben teilgenommen, die als Kunden der Verkehrsdienstleister oder als selbstfahrende Unternehmen (Werkverkehr) eine wichtige Rolle im städtischen Wirtschaftsverkehr spielen; hinzu kommt als zweite wichtige Gruppe das Verkehrsgewerbe, insbesondere Speditionen, Frachtführer, Paketdienste und die Eisenbahn. Die Beteiligung der Verlader aus Industrie und Handel ist eine zentrale Bedingung, denn hier befindet sich auch sehr häufig die Dispositions- und Entscheidungsmacht über die Transportabwicklung (Raum-Zeit-Fenster). Aus den Unternehmen sind die für Materialwirtschaft, Logistik und Unternehmensplanung zuständigen Entscheidungsträger in den Geschäftsführungen bzw. der Vorstandsebene relevant. Vor allem das Verkehrsgewerbe muss seiner heterogenen Struktur entsprechend sehr differenziert berücksichtigt werden; starke Grossbetriebe z. B. der Speditionen vermögen die Runde schnell zu dominieren, was der Ausgewogenheit der Diskussion schaden kann. Aus den Verwaltungen der drei Städte waren die querschnittsorientierten bzw. wirtschaftsnahen Arbeitsbereiche beteiligt, insbesondere die Stadtentwicklung, die Stadt- und Verkehrsplanung, die Ordnungsbehörden, die Ämter für Wirtschaftsförderung, ferner die Begleitforschung des Bundes und der Forschungsnehmer.

Eine wichtige Vorbedingung für die erfolgreiche "Ansprache» des unternehmerischen Umfeldes war die engagierte Unterstützung des Modellvorhabens durch die örtliche Industrie- und Handelskammer (IHK Wuppertal-SolingenRemscheid). Über den persönlichen Einsatz des Leiters der Verkehrsabteilung der IHK wurde ein wesentlicher Teil der Kontakte zu den Unternehmen und den Geschäftsführungen hergestellt. Der Verkehrsausschuss der IHK, die Verkehrsleiterkreise der verladenden Wirtschaft und persönliche Kontakte boten einen guten persönlichen Zugang zu den meisten Unternehmen. Die «offizielle» Kommunikation des Projekts mit den Unternehmen wurde durch einen Unternehmensberater hergestellt.

\section{Erfahrungen - Vor- und Nachteile der Güterverkehrsrunde}

Die Vorteile der Einrichtung von Güterverkehrsrunden liegen auf der Hand: die Kommune kann aktuelle Einschätzungen der Unternehmen, etwa bezüglich Standortbewertung, Erweiterungs- oder Verlagerungsabsichten, einschätzen, sie erfährt Probleme und Kritik von seiten der Betriebe aus erster Hand, was gegenüber einer tendenziell abgeschotteten Verwaltungspraxis einen erheblichen Wissensvorsprung mit sich bringen kann. Die Unternehmen bedienen sich ihrerseits zunächst einer Art «Feuerwehrfunktion» der Güterverkehrsrunde, indem sie über anstehende Planungen von den Kommunen - früher als dies allgemein geschieht - informiert werden oder diese mit Eingaben, Stellungnahmen etc. beeinflussen. Darüber hinaus kann es aber auch zur gemeinsamen Entwicklung von Lösungen kommen, die den Planungszielen der Kommune entsprechen.

Eine Kooperationsstrategie unter den Unternehmen - Voraussetzung nicht nur für Modelle der Stadtlogistik, sondern bspw. auch zur Anpassung der unterschiedlichen Zeitregimes zwischen Versender, Transporteur und Empfänger unabdingbar - kann dann leichter realisiert werden, wenn an den im Wortsinn entscheidenden Stellen Personen handeln, die sich kennen und ein im begrenzten Umfang vertrauenswürdiges Milieu existiert. Die Kooperationsbereitschaft der im Modellvorhaben beteiligten Unternehmen hat im Zeitverlauf deutlich zugenommen. Eine stärkere Bündelung nich konsolidierter Transporte in der Stadt wurde ausdrücklich als notwendiger und richtiger Schritt für die anstehende Massnahmenentwicklung bezeichnet, mehrere Betriebe wollten sich an konkreten Experimenten beteiligen. Auch der Einschätzung, dass für eine stärkere Koordination von Transporten Kompromisse von allen Seiten des Akteurssystems notwendig sind, wurde ausdrücklich zugestimmt. Dies sollte als politischer Erfolg des Vorhabens (und Anknüpfungspunkt für weitere Schritte) nicht unterschätzt werden, ganz unabhängig davon, dass dieser Effekt - auch in ökologischer Hinsicht-wohl kaum messbar ist.
In der Praxis werden aber auch die Nachteile bzw. Schwierigkeiten in der Handhabung von Güterverkehrsrunden deutlich. Zwischen theoretischer Debatte und praktischer Erprobung besteht noch eine grosse Diskrepanz - das Miteinander-Reden-Können muss nicht zwangsläufig auch ein Miteinander-HandelnKönnen bedeuten, so wie eine funktionierende Kommunikation bei der Mehrzahl der Betriebe nicht automatisch eine Bereitschaft zur praktischen Kooperation signalisiert. Kommunikation als Selbstzweck kann den mit der Güterverkehrsrunde verbundenen Aufwand aber nicht rechtfertigen. Ein diesbezügliches Problem "intermediärer» Einrichtungen liegt in der Zeitperspektive: ein daverhafter Erfolg lässt sich nur mit einer stabilen, relativ homogenen Zusammensetzung der Gruppe erzielen. Der dazu notwendige Zeitrahmen ist beträchtlich und wird bei weitem nicht der Dynamik gerecht, mit der sich die Abläufe in den Unternehmen, im Stadtverkehr etc. ändern. Die Beteiligung einzelner Unternehmen ist zudem im Regelfall an das Engagement interessierter einzelner Personen gebunden; verlassen diese den Betrieb, bricht nicht selten der Kontakt $\mathrm{ab}$.

Ein weiteres Problem dieser Verfahren liegt in ihrer Freiwilligkeit. Solange sich Kooperationsansätze nur auf wenige Unternehmen begrenzen und nicht allgemeinverbindlich sind, bleiben "spillover»-Effekte (das Überschwappen auf Nachbarfelder) naturgemäss begrenzt. Mit kommunikativen Strategien wird i.d.R. nur ein Teil des unternehmerischen Spektrums erreicht, so dass gleichzeitig immer auch formale, allgemeinverbindliche Regeln notwendig sein werden, um Akzeptanz und Wirkung konkreter Massnahmen zu erzielen. Diese beiden wichtigen Faktoren sollten daher durch eine Art "Doppelstrategie" gefördert werden, indem erstens die Betriebe die gemeinsame Entwicklung von Massnahmen unter Mitwirkung der Kommune vorantreiben, aber zweitens darüber hinaus auch städtebauliche Standards und Restriktionen zum Einsatz kommen, z. B. angepasste Nutzungszeiten, Fahrzeuggrössen etc. 
Dabei werden verschiedene konzeptionelle Herangehensweisen und Handlungsstrategien deutlich, die zum besseren Verständnis der Funktion von "Güterverkehrsrunden» beitragen können. Bisher ist die logistische Modernisierung durch einen sehr einseitigen "Innovationspfad" gekennzeichnet, der auf die informationstechnische Durchdringung des Raumes und die systematische Durchleitung von Transaktionskosten und Folgekosten an Dritte setzt, in diesem Fall vor allem von Verladern und Empfängern auf die Spediteure. Die damit verbundene erhöhte Durchlässigkeit des Raumes soll zur Verbesserung der Transporteffizienz und zur Realisierung zusätzlicher Wachstumspotentiale beitragen. Davon zu unterscheiden wäre ein Verständnis von Logistik als sozialer Innovation, die auf eine stärkere zwischenbetriebliche Abstimmung und Kooperation und das Zusammenspiel von Unternehmung und öffentlicher Hand setzt. Dieser zweite Pfad soll am Beispiel des Übergangs von der warenbezogenen zur raumbezogenen Güterversorgung - der sogenannten "CityLogistik» - und ihrer Einbettung in eine lokale Innovations- und Kooperationsstrategie näher erläutert werden.

\section{Kooperationsansätze in der Praxis - das Beispiel City-Logistik}

Das originäre Einsatzfeld von privat-öffentlichen und überbetrieblichen Kooperationen, die auf eine systematische Kommunikation angewiesen sind, ist die sogenannte "City-Logistik». Ausgangspunkt der City-Logistik ist die Überlegung, von der warenbezogenen Anlieferung über verschiedene Transportwege zu unterschiedlichen Zeiten (Status-Quo) zur raumbezogenen, gebündelten Belieferung von Teilgebieten einer Stadt/eines Stadtzentrums zu kommen. Bisher muss für ein gleichbleibend hohes bzw. tendenziell wachsendes Transportaufkommen in der Stadt eine zunehmende Zahl von Fahrzeugen eingesetzt werden. Da die Verdichtung der Liefertakte und die wachsende Zahl von Wettbewerbern, die gleichzeitig bzw. parallel den Stadtraum befahren, insgesamt zu einer sinkenden Auslastung der Fahrzeuge führen, wird das Verhältnis zwischen Gütermenge und Frachtraumangebot immer ungünstiger.

Der ungenutzte Frachtraum könnte besser ausgelastet werden, wenn eine gegebene Gütermenge - statt vier halbleere Fahrzeuge auf engstem Raum zu staven bzw. anschliessend einen hohen Anteil Leerfahrten zu erzeugen - über Routen verteilt wird, wie es im gewerblichen Verkehr üblich ist. Dieses Prinzip lässt sich von einzelnen Betrieben auch auf ganze Teilräume ausweiten, durch die Aufteilung von Liefergebieten unter den Transporteuren. Der Versuch, zwischen Angebot und Nachfrage an Laderaum zu vermitteln, stellt idealtypisch die richtige Schnittmenge aus ökonomischer und ökologischer Effizienz dar.

Er ist gleichzeitig aber aufgrund der Konkurrenzsituation der Unternehmen ein sehr sensibles, problematisches Unterfangen. Die meisten Betriebe haben gegen die mit einer solchen Bündelung zwangsläufig verbundenen "gläsernen" Strukturen erhebliche Vorbehalte. Unter den gegebenen Wettbewerbsbedingungen von Handel und Transportwirtschaft stellt sich ein solcher Eingriff in die Distributionsstrukturen noch als sehr schwierig dar und wird von den Nutzern nur bei hohen Risiken und Kosten des Status quo akzeptiert. Die Umsetzungshürde für solche Experimente ist noch sehr hoch. (Aus diesen Gründen ist auch ein entsprechender Feldversuch des Modellvorhabens nicht zustande gekommen). Es gibt insgesamt keine sachliche Grundlage für die Überlegung, City-Logistik "von oben» planen oder einführen zu wollen. Dies erklärt auch den Tatbestand, dass der inflationär angewachsenen Zahl von City-Logistik-«Konzepten» und -Ideen eine bisher nur geringe Zahl von realen Aktivitäten gegenübersteht (vgl. Hatzfeld, Hesse 1994). Vor dem Hintergrund des sehr heterogenen Interessengeflechts der städtischen Logistik ist vielmehr nach konkreten Anknüpfungspunkten zu suchen, die erste Schritte zur Optimierung der Belieferung ermöglichen und von denen dann weitergehende Impulse für die Innenstadtlogistik ausgehen können. Dabei kommt einer unternehmensübergreifenden Kom- munikation die wichtige Funktion des Impulsgebers zu.

Für die Umsetzung einer City-Logistik scheint ein stufenweises Vorgehen sinnvoll. Vor dem Hintergrund der jeweils spezifischen Kompetenzen und Zuständigkeiten der beteiligten Akteure sollte den privaten Unternehmen darin die Organisation und Umsetzung der City-Logistik zukommen, während die Kommune die geeigneten Rahmenbedingungen gestalten sollte, um Anpassungsreaktion der Betriebe - quasi selbstorganisiert in Gang zu setzen. Dieses arbeitsteiligregulative Vorgehen erscheint angemessener als der vergebliche Versuch der öffentlichen Hand, sich zum «ideellen Gesamtlogistiker" zu machen. In jedem Fall bedarf die City-Logistik aber einer aktiven Begleitung und Unterstützung durch öffentliche (hoheitliche) Massnahmen, da die einzelwirtschaftliche Optimierung bisher noch nicht dazu führt, dass definierte raumbezogene Wirkungen sichergestellt werden. Bei den notwendigen flankierenden Massnahmen handelt es sich um einen Mix aus verschiedenen Instrumenten, der positive Anreize - die Bevorzugung stadtverträglich organisierten Wirtschaftsverkehrs mit allgemeinverbindlichen Normen und Standards - Restriktionen für die nicht an entsprechenden Optimierungskonzepten beteiligten Unternehmen (Einsatz von push- und pull-Instrumenten). Diese Massnahmen sollten vor allem an sensiblen Teilräumen mit besonderer Problemdichte ansetzen.

Als spezifische, aber doch verallgemeinerbare soziale Erfolgsvoraussetzungen für eine Kooperation, die ausdrücklich durch die Einrichtung von Güterverkehrsrunden gefördert werden kann, gelten m. E. insgesamt die folgenden Faktoren:

- gegenseitiges persönliches Kennen und Vertrauen,

- gegenseitige Offenheit statt einseitiger Interessenvertretung,

- Verständigung über die Notwendigkeit von Planung statt «laissez-faire»,

- Verständigung über die Notwendigkeit never Lösungen (z.B. statt Anpassungsplanung)

- also: Kooperationswillen und -fähigkeit der Beteiligten auf allen Seiten. 
Eine besondere Bedeutung besitzt hierbei das lokale "Klima», in dem Massnahmen entwickelt werden bzw. Verkehrsentwicklung, Städtebau/Stadtplanung, Wirtschaftsförderung etc. diskutiert werden. Hier liegt eine weitere wichtige Vorbedingung für Kooperationen zwischen Unternehmen und Kommune sowie unter den Betrieben. Zum einen wird es in Zukunft stärker darauf ankommen, die zeitweise erhitzte Verkehrsdiskussion auf das Mass dessen zurückzuführen, was die Sachlage, die Interessenlage und die Gestaltungsoptionen vor Ort hergeben. Zum anderen wird es zu neven Kooperationsformen kommen müssen, wobei die Zusammenarbeit mit Unternehmen (nicht nur ihren Verbänden) einen zentralen Stellenwert einnehmen sollte. Schliesslich sollten dazu die schon vorhandenen Institutionen stärker eingebunden werden; die in der Region bisher mit den Schlüsselpersonen des örtlichen Handels erzielte Verständigung zum Thema Innenstadtlogistik hängt auch von den vorhandenen und erprobten Kommunikationsstrukturen ab (beispielsweise City-Management).

Zwei weitere wichtige Akteure dürfen hier nicht fehlen. Zum einen muss die Rolle des Staates als Rahmensetzer offensiv ausgeschöpft werden, wenn faire Wettbewerbsbedingungen und "wahre" Preise die Voraussetzungen zur Akzeptanz von lokalen und regionalen Massnahmen schaffen sollen. Bisher fehlt noch der notwendige Rahmen für eine stärker städtebaulich und sozial-ökologisch orientierte Verkehrspolitik. Anhaltender Druck auf die Preiskalkulation im Unternehmen (Verfall der Transportpreise insgesamt) sowie die stark verzerrte Kostensituation zwischen Schiene und Strasse (z. B. bei den Infrastrukturen) behindern immer noch eine stärkere Verlagerung bzw. Substitution von Transporten zwischen beiden Verkehrsträgern. Zum anderen stellt sich die Frage, inwieweit die sensible Diskussion um den Güterverkehr aus dem Unternehmen bzw. der Verwaltungspraxis in die allgemeine Öffentlichkeit hinein (z.B. gegenüber Anwohnern/-innen) geöffnet werden kann. Auch wenn der private Pkw-Verkehr einen wesentlich grösseren Anteil als der Wirtschaftsverkehr in der Stadt hat, so sind angesichts der spezifischen Belastungen vor allem durch die Güterbeförderung Entlastungen dringend erforderlich.

\section{Beiłräge zu einer ökologisch orientierten Unternehmensführung}

Der hier skizzierte Diskursansatz ist auch anschlussfähig an die Diskussion um eine ökologisch orientierte Unternehmensführung. Künftig werden sich die Unternehmen stärker mit den ökologischen, sozialen und gesellschaftspolitischen Folgen ihrer Produktionsweise und ihrer Produkte befassen müssen als bisher. Davon sind nicht nur die Verkehrswirtschaft oder die Automobilindustrie berührt, sondern alle auf Verkehrsdienstleistungen angewiesenen Unternehmen. Diese agieren auch in der Marktwirtschaft bekanntlich nicht allein in einer rationalen, ausschliesslich ökonomisch determinierten Sphäre, sondern sie stehen in engen Wechselbeziehungen zu Umwelt und Gesellschaft (vgl. Pfriem 1995). Umweltorientierung unternehmerischen Handelns steht nicht im luftleeren Raum, sondern kann sich auf eine nachprüfbare Veränderung von Teilen des Wertesystems der Gesellschaft stützen. Durch die verstärkte Wahrnehmung ökologischer Wirkungskreise werden auch die Unternehmen zunehmend in die umweltpolitische Pflicht genommen. Von den Betrieben, die bisher überwiegend Verkehrserzeuger waren, werden verstärkt auch Lösungsstrategien und ökologisches Know-how eingefordert (vgl. Spelthahn u. a. 1993).

Ein entscheidender Fortschritt wäre erreicht, wenn jene Perfektion, mit der die innerbetriebliche Logistik organisiert wird, auch für eine raumbezogene Transportorganisation nutzbar gemacht werden könnte. Hierzu gibt es bereits einige konkrete Ansatzpunkte, die auch die Rolle der öffentlichen Hand als Rahmensetzerin und Anreizgeberin berühren. Für die Umsetzung dieser Innovationen besitzt ein vorausschauendes, offensives Handeln des Managements zwar die Schlüsselrolle; das bereits vorhandene Umdenken einzelner Entscheidungsträger in den Betrieben muss aber durch die «zivilgesellschaftlichen» Akteure unterstützt werden. Neve strategische Allianzen zwischen (einzelnen) Unternehmen, den Kommunen und gesellschaftlich relevanten Kräften sollten die Rahmenbedingungen für ein derart ökologisch verpflichtetes Handeln der Innovateure innerhalb der Unternehmen verbessern. Güterverkehrsrunden könnten den wichtigen ersten Schritt hierzu leisten.

\section{Eine Zukunft für die Güter- verkehrsrunde?}

Die Güterverkehrsrunde im bergischen Städtedreieck hat ihre Arbeit nach Abschluss des Forschungsvorhabens beendet. Ihre 1991 verabredete Aufgabe wurde mit der Begleitung der Forschungstätigkeit und der Diskussion der Ergebnisse zunächst erfüllt. Eine mögliche, logische Konsequenz dieser Entwicklung könnte nun sein, den Diskurs einzustellen. Eine Alternative hierzu wäre der Gedanke, den Gesprächsfaden ggf. unter einer anderen Prämisse - wieder aufzunehmen und eine mehr oder minder kontinuierliche Kommunikation über Fragen des Güterverkehrs in der Stadt zu führen, die schliesslich auch institutionell abgesichert werden müsste.

In der letzten Sitzung der Güterverkehrsrunde wurden diese verschiedenen Konsequenzen im Anschluss an die Präsentation der Ergebnisse des Forschungsvorhabens diskutiert. Aus dem Kreis der Teilnehmer wurde überwiegend die Meinung vertreten, dass die Güterverkehrsrunde in der gegebenen Zusammensetzung und Zielsetzung ihren Zweck erfüllt habe und abgeschlossen werden sollte. Ausgehend von der insgesamt positiv beurteilten Wirkung der Runde wurde aber gleichzeitig die $\mathrm{Ab}$ sicht bekundet, die in Gang gesetzte Kommunikation weiterzuführen. Vor allem bezüglich der Themenbereiche Innenstadtbelieferung und City-Logistik wurde ein grosser Bedarf an Austausch von Positionen und Entwicklung von Strategien gesehen, wozu ein solches Diskussionsforum einen sinnvollen Rahmen bieten könnte.

Den stark ausdifferenzierten Interessenlagen im System des städtischen Gü- 
terverkehrs entsprechend, soll sich dieses Gremium mit Schwerpunkt konkreten Handlungsfeldern in konkreten Teilräumen widmen, nachdem das Forschungsvorhaben definitionsgemäss den Gesamtrahmen des städtischen und regionalen Güterverkehrs betrachtet hat. Die Industrie- und Handelskammer hat sich bereit erklärt, den organisatorischen Rahmen für einen solchen Arbeitskreis zu schaffen. Unter der Voraussetzung, dass in dieser Diskussion nicht nur betriebswirtschaftliche Ziele verfolgt werden, sondern auch das ökologische und städtebauliche Wirkungsspektrum gleichberechtigt behandelt wird, wäre ein solcher Schritt im Sinne einer Verstetigung der Zusammenarbeit von öffentlichen und privaten Akteuren nur zu begrüssen.

\section{Literatur}

Cooper, J.; Browne, M.; Peters, M. (1991): European Logistics. Markets, Management and Strategy. - Oxford

Grabher, G. (1994): Lob der Verschwendung, Berlin

Hatzfeld, U.; Hesse, M. (1994): Stadtlogistik Interessen statt Logistik? In: Internationales Verkehrswesen 46, H. 11, S. 646-653

Hatzfeld, U.; Hesse, M. (1993): Städtischer Wirtschaftsverkehr - die unbekannte Grösse. In: Städte- und Gemeinderat 7, S. 183-194

Hesse, M. (1994): Stadtverträglicher Wirtschaftsverkehr im bergischen Städtedreieck Remscheid-Solingen-Wuppertal, unveröff. Forschungsbericht, Wuppertal

Hesse, M. (1994): Industriebeziehungen und Innovationen. Neue Ansatzpunkte zur Verkehrsvermeidung im Güterverkehr, Diskussionspapier des Instituts für ökologische Wirtschaftsforschung (IÖW), Berlin

Hesse, M. (1993): Gute City-Logistik braucht den Runden Tisch. In: Deutsche Verkehrs-Zeitung (DVZ) vom 23.11.1993

Hesse, M. (1992): Verkehrswirtschaft auf neuen Wegen. Unternehmenspolitik vor der ökologischen Herausforderung, Marburg Pfriem, R. (1995): Unternehmenspolitik in sozial-ökologischen Perspektiven, Marburg

Selle, K. (1992): Planung im Wandel - Vermittlungsaufgaben und kooperative Problemlösungen, in: DISP 106, S. 34-45

Spelthahn, S.; Schlossberger, U.; Steger, U. (1993): Umweltbewusstes Transportmanagement, Bern 